\title{
Price, cost and value of opiate detoxification treatments
}

\author{
Reanalysis of data from two randomised trials
}

MICHAEL GOSSOP and JOHN STRANG

\author{
Background Treatments in different \\ settings have different costs. A dilemma \\ arises if expensive treatments lead to \\ better outcomes.
}

\begin{abstract}
Aims To investigate conflicts between the priorities of cost minimisation, clinical effectiveness, and cost-effectiveness in the detoxification of opiate addicts.
\end{abstract}

Method Cost and clinical effectiveness were examined using published outcome data. The main outcome measures were: achieving a drug-free state on completion of detoxification; the economic costs of treatment.

Results In terms of simple cost, inpatient detoxification is much more expensive than out-patient treatment (ratio, 24:I).With adjustment for successful outcome, the costs are almost identical (ratio, 0.9:I). Comparison of specialist and general psychiatry in-patient settings showed that even when adjusted for clinical outcomes, the specialist setting is more costly (ratio, 1.9:1), although the outcomes are better.

Conclusions Naïve adherence to cost and cost-containment considerations is dangerous. Discussion of treatment costs is misleading if not informed by, and adjusted for, evidence of effectiveness. This is especially important where marked differences in outcome between treatment options exist.

Declaration of interest Partial funding from the Medical Research Council.
The clinician seeks to deliver the most effective treatment for the individual patient. The purchaser seeks to maximise the health gains of the population with the available financial resources. The task of the manager is to deliver the product (e.g. occupied bed-days, treatment slots) as specified within the negotiated contract. The challenge for the patient (and advocates for the patient) is to secure the best treatment available from which they can benefit, regardless of the considerations of organisation and finance which may influence the decisions of others. Calculation of the types and extent of treatment benefit may be made separately, and in different ways, by the treatment purchaser, by agencies, by clinicians, and by the individual patient, and these calculations may lead to different conclusions. This paper examines the links, but also the tension and sometimes the conflict, that can exist between the priorities of cost minimisation, clinical effectiveness, and costeffectiveness (Honigsbaum et al, 1995).

\section{METHOD}

Previously published data from clinical research studies have been re-examined to calculate the costs of clinically important outcomes for patients who have been treated in different types of treatment settings. Two different elements of treatment programmes are investigated.

The first element we consider is the detoxification phase of treatment. Detoxification is designed and delivered with the specific purpose of reducing or eliminating withdrawal symptoms and helping the patient to achieve a drug-free state. Detoxification per se may be evaluated according to several outcome criteria, including severity of symptoms, duration of symptoms and rates of completion of the programme. In this study we use completion rates as the outcome measure against which costs are examined.

Detoxification outcome data are taken from the study of Gossop et al (1986). Detoxification completion rates were investigated in heroin-dependent patients assigned in a $2 \times 2$ design, either randomly or by patient's preference, to treatment in either a dedicated in-patient setting or a specialist drug-dependence out-patient clinic. The outcome criterion measure was achieving a state of abstinence from opiates for a minimum period of 24 hours at the completion of the programme. Successful detoxification completion rates of $81 \%$ were observed with patients treated in an in-patient drug-dependence unit and 17\% for patients treated in an out-patient drug-dependence clinic: these figures are used as examples of outcomes for patients treated in such settings, and cannot necessarily be generalised to the treatment of such patients in different settings, although they are consistent with the findings from a range of other studies we conducted in in-patient and out-patient settings with different groups of patients and using different specific treatment interventions (Gossop et al, 1989; Strang \& Gossop, 1990; Dawe et al, 1991; Unnithan et al, 1992).

The second type of treatment programme examined is the more comprehensive package in which detoxification is incorporated as a first phase, followed by a range of psychological and relapseprevention treatment interventions. Outcome data for patients treated in a specialist in-patient unit were compared with data for patients treated on a general psychiatric ward (Strang et al, 1997). Opiate addicts seeking in-patient treatment were randomly assigned to one of the two in-patient settings in the same hospital. Outcome data were presented for different denominator samples (original randomised cohort; those entering treatment; those who completed detoxification; etc.) with follow-up of a subsample at 2 months and at 7 months. The successful detoxification completion rates were $45 \%$ and $18 \%$ of the original randomised cohort for the specialist inpatient unit and the general psychiatric ward respectively; or, if the analysis is restricted to only those patients who subsequently entered the in-patient wards, $75 \%$ and $43 \%$ respectively.

Longer-term outcome data were also available for a subsample at 2 months and at 7 months after treatment: $65 \%$ of the 
specialist treatment sample and $23 \%$ of the general psychiatric treatment sample were abstinent at 2 months, $79 \%$ and $31 \%$ of the samples, respectively, at 7 months. If the less stringent outcome criterion of avoidance of daily opiate use is considered, then at 2 months improvement is seen in $80 \%$ of the specialist treatment sample and $61 \%$ of the general psychiatric treatment sample; at 7 months, the comparable figures are $83 \%$ and $46 \%$ respectively. More detailed descriptions of the treatment interventions and the two treatment settings are published elsewhere (Powell et al, 1993; Strang et al, 1997). Such outcome data for these treatment-seeking opiate addicts are broadly consistent with the follow-up data at 2 months and 6 months previously reported in the post-detoxification Maudsley Relapse Study (Bradley et al, 1989; Gossop et al, 1989).

Calculation of the costs for these treatments was not undertaken at the time of these original studies. However, these inpatient and out-patient services were subsequently involved in the National Treatment Outcome Research Study (NTORS), as one of the sites for the inpatient and out-patient samples. Their costs were therefore calculated to enable the costs within NTORS to be evaluated. These data were collected by the Centre for the Economics of Mental Health from the 54 participating residential and community agencies as part of an investigation of the economic costs of treatment (Healy et al, 1998).

Calculations of the costs of the inpatient and out-patient detoxification programmes are made on the basis of both mean and median programme costs. Several cost calculations are carried out. For the first comparison, these are: the cost per week; the cost per full treatment episode (calculated for a 21-day in-patient detoxification, for a 10-day in-patient detoxification, and for a 6-week out-patient programme); and the cost per successful, abstinent case. For the second comparison, the in-patient drug dependence unit and general psychiatry costs were calculated for: cost per week; cost per episode, using the actual mean lengths of stay of 69 and 24 days respectively (Strang et al, 1997); the cost per abstinent case (i.e. completed detoxification); and the costs adjusted both for abstinence and for a less stringent criterion of clinical improvement (abstinence or reduced frequency of heroin use) at 2-month and 7-month follow-ups.
The calculation of costs adjusted for outcomes was made using the equation:

$$
\frac{\text { cost per week } \times \text { duration }}{\text { outcome }} \text {. }
$$

The outcome rates were taken directly from the research findings of Gossop et al (1986) and Strang et al (1997). These are $81 \%$ for completion of in-patient detoxification, and $17 \%$ for completion of out-patient detoxification. For the comparison of drug dependence unit and general psychiatry in-patient programmes, they are: abstinence at 2 months, $65 \%$ and $23 \%$ respectively; improvement at 2 months, $80 \%$ and $61 \%$; abstinence at 7 months, $79 \%$ and $31 \%$; and improvement at 7 months, $83 \%$ and $46 \%$.

\section{RESULTS}

\section{In-patient v. out-patient detoxification}

The costs of detoxification provided in an in-patient setting are very much higher than those of detoxification delivered in an outpatient setting (see Table 1). The difference is greatest for simple, unadjusted costs per week where, within the limitations of the costing model used, we find the cost of the in-patient detoxification to be 24 times that of the out-patient treatment. The difference is still clearly evident when comparisons are made of the full detoxification treatment packages. In our calculations, the costs of a 3-week in-patient detoxification programme are nine times those of an 8-week out-patient programme.

A more informative calculation of the relative cost-effectiveness of the detoxification in the two settings can be made by adjusting the costs of the treatment packages according to their observed success rates. The costs of the in-patient detoxification programme should therefore be divided by 0.81 to adjust for the $81 \%$ abstinence rate of patients treated in this programme, and the costs of the out-patient programme could be divided by 0.17 (Gossop et al, 1986). When adjusted for successful achievement of abstinence, the difference is greatly reduced-the inpatient treatment costs less than twice as much as the out-patient treatment.

During the past decade, in-patient detoxification has increasingly been provided in shorter programmes than the 21day programme described by Phillips et al (1987). Frequently, detoxification is now provided over a 10-day period, and it has been shown that 10-day detoxification programmes can be as effective (in terms of reducing withdrawal severity and of retaining patients in treatment to the point of abstinence) as the longer 21-day programme (Gossop et al, 1989; Gossop \& Strang, 1990; Bearn et al, 1996, 1998). In-patient opiate detoxification is now seldom delivered over periods as long as 21 days, and 10 days is frequently the standard for current in-patient detoxification. For this reason, additional calculations were made for the cost of each abstinent case within a 10-day in-patient programme. These costs are shown in Table 1 and are indicated as 'Cost per abstinent episode' (line 5). In contrast to the direction of the difference seen with the cruder cost calculations, the cost per abstinent case with the 10-day in-patient detoxification was slightly less than the cost per abstinent case in the out-patient programme.

\section{In-patient treatment in a specialist drug dependence unit v. treatment in a general psychiatry setting}

The costs of treatment in a dedicated inpatient drug dependence unit (DDU) and in a general psychiatry in-patient ward are

Table I Comparative costs and cost outcomes of detoxification in specialist settings

\begin{tabular}{lccc}
\hline Cost & $\begin{array}{c}\text { In-patient } \\
(\ell)\end{array}$ & $\begin{array}{c}\text { Out-patient } \\
{ }^{3}\end{array}$ & Ratio $\left.^{(}\right)$ \\
\hline Per week & 927.4 & 39.1 & $24: 1$ \\
Per episode' & 2782 & 313 & $9: 1$ \\
Per abstinent ${ }^{4}$ case $^{1}$ & 3435 & 1840 & $2: 1$ \\
Per episode $^{2}$ & 1325 & 313 & $4: 1$ \\
Per abstinent $^{4}$ case $^{2}$ & 1636 & 1840 & $0.9: 1$ \\
\hline
\end{tabular}

I. Figures calculated for a $2 \mathrm{I}$-day in-patient detoxification programme.

2. Figures calculated for a 10 -day in-patient programme.

3. Figures for out-patient detoxification are calculated for a 6 -week programme.

4. In this context, an abstinent case is taken to be one who has successfully completed detoxification. 
shown in Table 2, and are then adjusted to provide the costs for outcomes. The simple costs per week could generally be considered to be similar, although the costs of treatment episodes in the two settings differ (because of the markedly different lengths of stay), with costs per episode on the DDU being about three times those of treatment on the general psychiatry ward. The costs per successfully completed detoxification have also been calculated, thus taking into account the different detoxification outcomes on the two wards: purchase of a successful detoxification on the specialist DDU ward remains more expensive, although the cost differential has by now come down to 1.9:1.

Further analyses of these data have been conducted, to look at the purchase of successful outcome at follow-up. When adjusted for abstinent outcomes at 2 months, the costs are similar for the two treatment settings (see Table 3). When adjusted for the markedly different outcome rates (measured first as abstinence, and then according to the more stringent criterion of opiate use reduced to occasional or irregular use), the costs again diverge, the costs of the dedicated unit being more than twice those of the general psychiatry ward. The costs adjusted for abstinence at 7 months are similar, although slightly greater for the DDU, and the costs for improved outcomes at 7 months are higher for the DDU.

Costs can also be calculated for the most stringent outcome: continuous abstinence throughout the 7-month follow-up period. This calculation can be made only for patients who were treated in the DDU, since no patients treated in the general psychiatry ward were able to maintain abstinence throughout this period. This provides the clearest difference in outcome between the treatments and on this basis, only the DDU was cost-effective. Forty two per cent of the DDU patients were continuously abstinent, and the cost of continuous abstinence over the 7-month follow-up period for these patients is $£ 21767$ (calculated as $£ 9142$ divided by 0.42 ).

\section{DISCUSSION}

\section{Relative costs of treatment}

One of the most conspicuous differences between a treatment intervention delivered in an in-patient or in an out-patient setting is that of cost. In our calculations, the unadjusted weekly cost of an in-patient detoxification is 24 times that of an outpatient detoxification. Even when adjustment is made for the possibility that the treatment of the withdrawal syndrome may be achieved more rapidly in an inpatient setting, the crude costs are still much higher than those for out-patient treatment. When adjustments are made for outcome, the differences in cost become less marked. The costs of a 10-day inpatient programme, when adjusted for the different rates at which patients successfully completed the programme, are almost identical to those of the out-patient programme (indeed, they are marginally lower). Similarly, a lengthier stay on a specialist in-patient unit was over three times more expensive than brief admission to a general psychiatry ward, and remained nearly twice as expensive after correction for different short-term outcomes. Even when examining medium-term outcomes of either abstinence or improvement, and correcting accordingly, the specialist treatment was typically still twice as costly, despite the better outcomes.

\section{Considerations beyond cost}

Discussion of treatment costs can be misleading if it is not informed by, and adjusted for, evidence of effectiveness. This is especially important where there are marked differences in outcome between treatment options. For some time, and in many countries, existing treatment services have been exposed to budget limitations and management pressures which have relied heavily upon the terminology of cost-effectiveness but which have often placed greater weight upon accountancy administration or upon the simple issue of costs. Too much emphasis has been placed upon the simple financial or accounting aspects. Over-reliance on cost issues is a misunderstanding of the aims of costeffectiveness studies, and should be more accurately referred to as cost minimisation (Lockett, 1997) or cost-containment. In the United States, Swift \& Miller (1997) have described how radical changes have occurred in the availability and funding of addiction treatment services, so that inpatient treatments and treatments in residential settings are largely being replaced by community-based treatment services, with clinical decisions being directly influenced by insurance coverage rather than severity of illness.

In addition, the characteristics and problems of drug misusers who seek treatment in out-patient services are known to differ from those treated in in-patient services. In general, the more difficult cases tend to be treated in residential programmes (Gossop et al, 1998). These sorts of case-mix issues are further factors which

Table 2 Comparative costs and cost outcomes of addiction treatment in in-patient settings

\begin{tabular}{lccc}
\hline Cost & $\begin{array}{c}\text { Drug dependence unit } \\
\end{array}$ & $\begin{array}{c}\text { General psychiatry } \\
(\ell)\end{array}$ & Ratio \\
\hline Per week & 927.4 & 805.3 & $1.2: 1$ \\
Per episode & 9142 & 2761 & $3.3: 1$ \\
Per abstinent case (i.e. completed detoxification) & 12189 & 6421 & $1.9: 1$ \\
\hline
\end{tabular}

The figures given represent mean treatment costs.

Calculations based on completion rates for the patients admitted (see text).

Table 3 Comparative costs and cost outcomes at follow-up of addiction treatment in specialist and general psychiatry settings

\begin{tabular}{lccc}
\hline Cost & $\begin{array}{c}\text { Drug dependence unit } \\
\text { Foneral psychiatry }\end{array}$ & Ratio \\
\hline For abstinence at 2 months & $(£)$ & $(£)$ & \\
For improvement at 2 months & 14065 & 12004 & $1.2: 1$ \\
For abstinence at 7 months & II 428 & 4526 & $2.5: 1$ \\
For improvement at 7 months & II 572 & 8906 & $1.3: 1$ \\
\hline
\end{tabular}

The figures given represent mean treatment costs. 
must be taken into account in the decision about how, and in what ways, effective treatment services should be provided.

A further illustration of the manner in which the adjustment of costs is required to take account of outcome differences is provided in our second comparison, between in-patient treatment provided in a dedicated DDU and on a general psychiatry ward. In this case, the difference in unadjusted costs per week is less marked than that between in-patient and out-patient settings. However, the duration of treatment in these two in-patient settings was such that when adjustment was made for the actual length of stay, the cost of a treatment episode on the DDU was about three times that of one on the general psychiatry ward. When adjusted for different clinical outcomes, the cost difference drops considerably, although the DDU continues to be the more costly of the two options. For individual patients, however, the outcomes of treatment on the DDU are consistently superior.

Our data and analyses illustrate the dangers of naïve adherence to cost and costcontainment considerations. Provision of in-patient detoxification in a non-specialist setting will undoubtedly be easier to establish, and probably cheaper to run, than in a specialist service. Provision of only out-patient detoxification offers great opportunities for substantial cost-cutting but at a huge cost to individual patients, their families and society. When the substantial differences in immediate outcomes (successful completion of detoxification) are included in the consideration, the costs of purchase of successful detoxification in the different settings become more comparable - and even more so when considering the purchase of successful postdetoxification abstinence in the follow-up period. When the wider costs to society are included in the consideration, the inappropriateness of a naïve cost minimisation approach becomes even more evident. Some types of problem behaviour displayed by drug addicts create heavy costs for the health and criminal justice systems (Gossop et al, 1997, 1998). In Canada, the economic costs of illicit drug use have been estimated to be about $\$ 1.4$ billion per year (Single et al, 1998). Current estimates put UK costs to the criminal justice system alone at more than $£ 1$ billion per year (Central Drugs Coordinating Unit, 1998). In this context, the logic of purchasing a cheaper detoxification programme with

\section{CLINICAL IMPLICATIONS}

- Treatment costs can be misleading if not informed by, and adjusted for, evidence of effectiveness: the cost-effectiveness of programmes which appear to differ greatly in cost at the point of provision can be very similar when adjusted for outcomes.

- Service planning decisions have frequently overemphasised cost containment with a focus upon simple financial or accounting issues: 'outcome-funding' or 'outcomepurchase' may be a more efficient way to commission drug misuse treatment services.

Prioritisation of cost containment will result in some patients being denied access to the necessary treatment and many patients being forced to accept suboptimal treatment.

\section{LIMITATIONS}

Many of the data in our calculations are based on detoxification treatments: consideration of longer-term outcomes is required.

- Crude costings are used, based on average costs: results might differ if costed for individual cases.

- The data are taken from services in a London teaching hospital and the findings may not generalise to other settings.

MICHAEL GOSSOP, PhD, JOHN STRANG, MRCPsych, National Addiction Centre, London

Correspondence: Professor M. Gossop, National Addiction Centre, 4 Windsor Walk, London SE5 8AF, UK

(First received 29 September 1999, final revision 23 February 2000, accepted 24 March 2000)

significantly worse completion rates becomes difficult to understand.

\section{Searching for a way forward}

Whose view should be afforded most priority? The clinician, the purchaser, the manager/administrator or the patient? Which outcomes should carry most weight? According to whose view is taken, which outcomes are considered important, and over what period, the calculated costeffectiveness of the different treatments can be regarded in different ways. Ultimately we should not forget that patients will still rightfully pursue the treatment with the best outcome for themselves, regardless of others' broader strategic considerations of health purchase across the wider population.

The patient's own interests (and those of family and other advocates for the patient) may be impossible to reconcile with the crude calculations which are based solely upon cost. It is unfortunate, therefore, that these are the calculations which often appear to be made by treatment purchasers and planners. For individual patients, the probabilities of outcome for their own treatment are paramount. It is little consolation to be told of the greater public health gain from the more widespread provision of care if the price to be paid is allocation to a treatment modality with sub-optimal outcome. The patient purchasing private health care would consider this an unacceptable argument, and the patient receiving National Health Service care should be similarly dissatisfied. As Cochrane (1972) noted, an ineffective service is inefficient and cannot be costeffective, no matter how cheaply it is provided. An 'outcome funding' or 'outcome purchase' approach may be a more efficient way of handling the commissioning of health care in this area. If health purchasers 
were to purchase successful outcome, then this would provide incentives to the care providers to make best use of the available resources: if longer in-patient care or more expensive specialist care were associated with sufficiently better outcome, then the clinician, administrator and purchaser would all be pursuing objectives similar to those which interest the patient.

\section{ACKNOWLEDGEMENTS}

The authors thank Professor Christine Godfrey from the Department of Health Sciences and Clinical Evaluation at the University of York, for her helpful comments on a draft of this paper. The second of the reference studies was originally supported by a Medical Research Council research grant.

\section{REFERENCES}

Bearn, J., Gossop, M. \& Strang, J. (1996) A

randomised double-blind comparison of lofexidine and methadone in inpatient treatment of opiate withdrawal. Drug and Alcohol Dependence, 43, 87-91.

_ , _ \& _ (1998) Accelerated lofexidine treatment regimen compared with conventional lofexidine and methadone treatment for inpatient opiate detoxification. Drug and Alcohol Dependence, 50, 227-232.

Bradley, B. P., Phillips, G., Green, L., et al (1989) Circumstances surrounding the initial lapse to opiate use following detoxification. British Journal of Psychiatry, 154 354-359.
Central Drugs Coordinating Unit (1998) Tackling Drugs to Build a Better Britain: The Government's 10-Year Strategy for Tackling Drug Misuse. London: HMSO

Cochrane, A. (1972) Effectiveness and Efficiency. London: Nuffield Provincial Hospitals Trust.

Dawe, S., Gossop, M., Griffiths, P., et al (199I) Should opiate addicts be involved in controlling their own detoxification? A comparison of fixed versus negotiable schedules. British Journal of Addiction, 86, 977-982.

Gossop, M., Johns, A. \& Green, L. (1986) Opiate withdrawal: inpatient versus outpatient programmes and preferred versus random assignment to treatment. British Medical Journal, 293, 103-104.

__, Green, L., Phillips, G., et al (1989) Lapse, relapse and survival among opiate addicts: a prospective followup study. British Journal of Psychiatry, 154, 348-353.

_, Griffiths, P., Bradley, B., et al (1989) Opiate withdrawal symptoms in response to 10 -day and 21 -day methadone withdrawal programmes. British journal of Psychiatry, 154, 360-363.

_ \& Strang, J. (1990) A comparison of the withdrawal responses of heroin and methadone addicts during detoxification. British Journal of Psychiatry, 158 697-699.

\section{_ , Marsden, J., Stewart, D., et al (1997) The}

National Treatment Outcome Research Study in the United Kingdom: six month follow-up outcomes. Psychology of Addictive Behaviors, II, 324-337.

$\ldots, \ldots, \ldots$, et al (1998) Substance use, health and social problems of service users at 54 drug treatment agencies. Intake data from the National Treatment Outcome Research Study. British Journal of Psychiatry, 173, $166-17 \mid$.

Healy, A., Knapp, M., Astin, J., et al (1998) The economic burden of drug addiction: social costs incurred by clients at intake to the National Treatment Outcome
Research Study (NTORS). British Journal of Psychiatry, 173, 160-165.

Honigsbaum, F., Richards, J. \& Lockett, T. (1995) Priority Setting in Action: Purchasing Dilemmas. Oxford: Radcliffe Medical Press.

Lockett, T. (1997) Evidence-based and Cost-effective Medicine for the Uninitiated. Oxford: Radcliffe Medical Press.

Phillips, G. T., Gossop, M. \& Bradley, B. (1987) An investigation of withdrawal symptoms shown by opiate addicts during and subsequent to a 21-day inpatient methadone detoxification procedure. Addictive Behaviors, 12, I-6.

Powell, J., Dawe, S., Richards, D., et al (1993) Can opiate addicts tell us about their relapse risk? Subjective predictors of clinical prognosis. Addictive Behaviors, 18 473-490.

Single, E., Robson, L., Xie, X., et al (1998) The economic costs of alcohol, tobacco and illicit drugs in Canada, 1992. Addiction, 93, 991-106.

Strang, J. \& Gossop, M. (1990) Comparison of linear versus inverse exponential methadone reduction curves in the detoxification of opiate addicts. Addictive Behaviors, I5, 54I-547.

-, Marks, I., Dawe, S., et al (1997) Type of hospita setting and treatment outcome with heroin addicts. Results from a randomised trial. British Journal of Psychiatry, I7I, 335-339.

Swift, R. M. \& Miller, N. S. (1997) Integration of health care economics for addiction treatment in clinic care. Journal of Psychoactive Drugs, 29, 255-262.

Unnithan, S., Gossop, M. \& Strang, J. (1992) Factors associated with relapse among opiate addicts in an outpatient detoxification programme. British Journal of Psychiatry, 161, 654-657. 